\title{
Regional trends and the impact of various patient and hospital factors on outcomes and costs of hospitalization between academic and nonacademic centers after deep brain stimulation surgery for Parkinson's disease: a United States Nationwide Inpatient Sample analysis from 2006 to 2010
}

\author{
Mayur Sharma, M.D., M.Ch., Sudheer Ambekar, M.D., M.Ch., \\ Bharat Guthikonda, M.D., Jessica Wilden, M.D., and Anil Nanda, M.D., M.P.H. \\ Department of Neurosurgery, Louisiana State University Health Sciences Center, Shreveport, Louisiana
}

\begin{abstract}
Object. The aim of this study was to analyze the incidence of adverse outcomes, complications, inpatient mortality, length of hospital stay, and the factors affecting them between academic and nonacademic centers after deep brain stimulation (DBS) surgery for Parkinson's disease (PD). The authors also analyzed the impact of various factors on the total hospitalization charges after this procedure.

Methods. This is a retrospective cohort study using the Nationwide Inpatient Sample (NIS) from 2006 to 2010. Various patient and hospital variables were analyzed from the database. The adverse discharge disposition and the higher cost of hospitalization were taken as the dependent variables.

Results. A total of 2244 patients who underwent surgical treatment for PD were identified from the database. The mean age was $64.22 \pm 9.8$ years and $68.7 \%(\mathrm{n}=1523)$ of the patients were male. The majority of the patients was discharged to home or self-care $(87.9 \%, \mathrm{n}=1972)$. The majority of the procedures was performed at high-volume centers $(64.8 \%, \mathrm{n}=1453)$, at academic institutions $(85.33 \%, \mathrm{n}=1915)$, in urban areas $(\mathrm{n}=2158,96.16 \%)$, and at hospitals with a large bedsize $(86.6 \%, \mathrm{n}=1907)$ in the West or South. Adverse discharge disposition was more likely in elderly patients $(\mathrm{OR}>1, \mathrm{p}=0.011)$ with high comorbidity index (OR 1.508 [95\% CI 1.148-1.98], $\mathrm{p}=0.004)$ and those with complications (OR 3.155 [95\% CI 1.202-8.279], $\mathrm{p}=0.033$ ). A hospital with a larger annual caseload was an independent predictor of adverse discharge disposition (OR 3.543 [95\% CI 1.781-7.048], p < 0.001), whereas patients treated by physicians with high case volumes had significantly better outcomes $(\mathrm{p}=0.006)$. The median total cost of hospitalization had increased by $6 \%$ from 2006 through 2010. Hospitals with a smaller case volume (OR 0.093, p < 0.001), private hospitals (OR 11.027, p < 0.001), nonteaching hospitals (OR 3.139, p = 0.003), and hospitals in the West compared with hospitals in Northeast and the Midwest (OR 1.885 [p = 0.033] and OR 2.897 [p= $0.031]$, respectively) were independent predictors of higher hospital cost. The mean length of hospital stay decreased from 2.03 days in 2006 to 1.55 days in 2010 . There was no difference in the discharge disposition among academic versus nonacademic centers and rural versus urban hospitals $(\mathrm{p}>0.05)$.

Conclusions. Elderly female patients with nonprivate insurance and high comorbidity index who underwent surgery at low-volume centers performed by a surgeon with a low annual case volume and the occurrence of postoperative complications were correlated with an adverse discharge disposition. High-volume, government-owned academic centers in the Northeast were associated with a lower cost incurred to the hospitals. It can be recommended that the widespread availability of this procedure across small, academic centers in rural areas may not only provide easier access to the patients but also reduces the total cost of hospitalization.

(http://thejns.org/doi/abs/10.3171/2013.8.FOCUS13295)
\end{abstract}

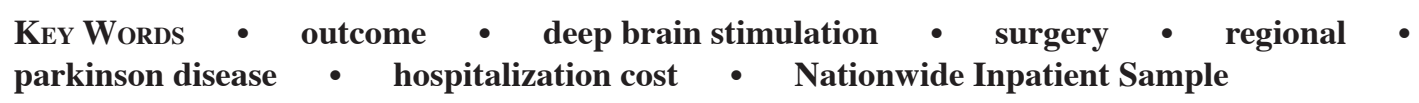

$\mathrm{B}$ UCY and Case proposed the first surgical treatment for Parkinson's disease (PD) in 1939 when they excised the motor cortex to relieve tremors associated with this condition. ${ }^{5}$ However, with the introduction of levodopa therapy in 1967, the surgical treatment of PD

\footnotetext{
Abbreviations used in this paper: DBS = deep brain stimulation; LOS = length of stay; NIS = Nationwide Inpatient Sample; OTR = other than routine; $\mathrm{PD}=$ Parkinson's disease.
}

was largely abandoned. ${ }^{7,8}$ With an improved understanding of the basal ganglia pathophysiology and a better control of all motor symptoms of PD, pallidotomy was successfully reintroduced in 1992 for the management of drug-resistant or refractory PD. ${ }^{15,16}$ With the advent of deep brain stimulation (DBS) for PD in the last decade, lesional surgery has again fallen out of favor, and DBS has become the treatment of choice for refractory or advanced PD. Reversibility of side effects and the ability 
to treat bilaterally and to optimize the treatment during programming are the key advantages of DBS over lesioning. ${ }^{14,20}$

Other indications of DBS are dystonia and tremor in movement disorders, depression, obsessive-compulsive disorder, and Tourette's syndrome in psychiatry, epilepsy, cluster headache, pain from stroke, amputation, trigeminal neuralgia, and multiple sclerosis..$^{1,12,17,21}$ The targets for DBS electrode implantation include globus pallidus internus and subthalamic nucleus for the treatment of refractory PD, pedunculopontine nucleus for the management of gait disorder in PD, the ventral intermediate nucleus of the thalamus for refractory essential tremors and globus pallidus internus for the treatment of dystonia. ${ }^{10,14,20,21,27,28}$

Being a widely used treatment modality, it is important to understand the various patient and hospital-related factors that can affect outcome after DBS surgery for PD. It is also prudent to be aware of the practice patterns and regional variations associated with this procedure. To address these issues, we analyzed the Nationwide Inpatient Sample (NIS) to study the outcomes and socioeconomic trends of the DBS procedure for PD. Eskandar et al. showed that the large-volume hospitals had superior short-term outcomes and lower total cost of hospitalization following DBS surgery for PD, using the nationwide sample from 1996 to $2000 . .^{10}$ The aim of our study was to analyze the incidence of adverse outcomes, complications, inpatient mortality, length of hospital stay, and the factors affecting them between academic and nonacademic centers after DBS surgery for PD using the US NIS from 2006 to 2010. We also analyzed the impact of various factors on the total hospitalization charges after this procedure.

\section{Methods}

\section{Data Source and the Study Sample}

Data were obtained from the NIS database, which is provided by the Healthcare Cost and Utilization Project (HCUP) Agency For Healthcare Research and Quality (AHRQ). ${ }^{9,25}$ This database represents an approximately $20 \%$ stratified sample of nonfederal community hospitals in the US. The NIS is the largest all-payer inpatient care database and contains data on 5-8 million hospital admissions from about 1000 hospitals in 45 states.

The data for PD from 2006 to 2010 were extracted from the NIS using the ICD-9 CM (International Classification of Diseases, 9th revision, Clinical Modification) diagnosis and treatment codes. In concordance with a previous study, all admissions with a primary diagnosis code of 332.0 (PD) or 332.1 (secondary parkinsonism) were included only when they were accompanied by the primary procedure codes for thalamotomy (ICD-9 CM code 01.41), pallidotomy (ICD-9 CM code 01.42), or implantation of a neurostimulator (ICD-9 CM code 02.93). ${ }^{10}$ Patients who underwent stereotactic radiosurgery (ICD-9 CM codes 92.30-92.33 and 92.39) for PD were excluded from this study, although this proportion constituted only $0.13 \%$ of all surgical admissions.

\section{Patient and Hospital Characteristics}

Patient Characteristics. Patient age, race, sex, primary payer (Medicare, Medicaid, private insurance, selfpay, no charge, or other), type of admission (emergency, urgent, or elective), day of admission (weekend/weekday), and median household income for patient ZIP code (ZIPINC_QRTL) were extracted directly from the NIS.

The following variables were recoded into different categories to further aid in our analysis: 1) Patient age was categorized into $\leq 65$ years and $>65$ years. 2) Race was recoded as white, black, and other for the statistical analysis. This variable was missing in $22.9 \%$ of admissions. 3) Comorbidities were classified using the Charlson's Index and were dichotomized (low comorbidity [0 or 1] vs high $[\geq 2]$ ) for the analysis. ${ }^{6}$ ) Specific complications following the surgical treatment of patients with PD were extracted using the following ICD-CM diagnosis codes: a) postoperative infarction/hemorrhage (997.00-997.02 and 997.09); b) postoperative bleeding/hematoma/seroma complicating a procedure (998.1 and 998.11-998.13); c) mechanical complication of nervous system device, implant, and graft (996.2); d) infections due to nervous system device, implant, and graft (996.63); and e) removal of intracranial neurostimulator lead (01.22). Patients were then grouped into those with and without complications. 5) Type of admission was recoded as elective and nonelective for the analysis. Missing values in the variables were not taken into consideration for the analysis.

Hospital Characteristics. Hospital region (Northeast, Midwest, South, or West), hospital location (rural or urban), teaching status, hospital bed size (small, medium, or large), and hospital control (government, private nonprofit, private, and investor owned) were coded in the NIS database. Hospital control was recoded into government and private for analysis. The number of cases at each hospital (hospital volume) was extracted from the NIS using the hospital identification number (HOSPID). Percentiles were calculated, and hospitals with case volumes greater than the 75th percentile were categorized into high-volume centers and recoded as high- and low-volume centers for analysis. Similarly, the number of surgically managed cases of PD for each surgeon (where available) were extracted from the NIS and were grouped into quartiles for analysis (1st quartile, 1 case; 2nd quartile, 2-3 cases; 3rd quartile, 4-12 cases; and 4th quartile, 13 or more cases). Both hospital and surgeon volume of cases showed significant positively skewed distribution, and therefore the logistic regression model was used while evaluating these variables for analysis.

\section{Outcome Factors}

The primary adverse end points in our study were discharge disposition to facilities other than home/selfcare (DISPUniform), inhospital mortality, hospital length of stay (LOS), complications, and total hospital charges. As mentioned in the NIS, discharge disposition to home was considered as routine and discharges to all other places (transfer to short-term hospital, skilled nursing facility, intermediate care facility, another type of facil- 


\section{Outcomes after DBS surgery for Parkinson's disease}

ity, home health care, against medical advice, and death) were recoded as other than routine (OTR) disposition. The total cost of hospitalization was extracted using the variable total charges (TOTCHG) in the NIS, and percentiles were calculated. The total cost of hospitalization greater than the 75 th percentile $(\$ 75,612.00)$ was recoded as high cost and the rest as low cost $(<75$ th percentile) for the analysis. Hospital LOS was also extracted from the NIS and was stratified according to the calendar year and the hospital region.

\section{Statistical Analysis}

Statistical Analysis was carried out using SPSS (version 21, IBM, Inc.) and Microsoft Excel. The chi-square and Pearson's correlation tests were used to evaluate the association between the categorical variables. For nonparametric analysis, the Kruskal-Wallis test was used to compare the mean total hospital charges across the different categories of ordinal data. Comparisons were considered significant only if the $\mathrm{p}$ value was $<0.05$. Univariate analysis was used to identify the covariates that might affect the outcome. Binary logistic regression analysis was used for the multivariate analysis to generate the probability value, odds ratio, and confidence interval. Two-tailed probability values were recorded. Variables with $\mathrm{p}<0.1$ in the univariate analysis were considered for the multivariate analysis. Discharge disposition, hospital charges, and inhospital mortality were the dependent variables used in the analysis.

\section{Results}

Of 14,291 admissions with a diagnosis of PD in the database from 2006 to 2010, 2244 (15.7\%) patients underwent surgical treatment of PD. Of these 2244 patients, only $5(0.2 \%)$ had a diagnosis of secondary PD. Implantation of the intracranial neurostimulator was the most frequently used procedure in $99.3 \%(n=2228)$ of the surgically treated patients. Other rare procedures such as thalamotomy, pallidotomy, and stereotactic radiosurgery were used in $0.3 \%(n=7), 0.3 \%(n=6)$, and $0.13 \%(n=3)$ of the patients. The clinical characteristics of the patients who underwent surgical treatment of PD are shown in Table 1 . The mean patient age was $64.22 \pm 9.8$ years (range 23-92 years); $49.6 \%(n=1108)$ of the patients were older than 65 years. Overall, 68.7\% $(n=1523)$ of the patients were male, and white was the dominant race $(64.8 \%, \mathrm{n}$ $=1454)$. Medicare was the primary payer for $62.8 \%(\mathrm{n}=$ 1405 ) of the patients, whereas private insurance was the primary payer for only $32.1 \%(\mathrm{n}=718)$ of the cases. For median household income for the ZIP code, $18.8 \%(\mathrm{n}=$ $411)$ and $28.4 \%(n=622)$ of the patients were in the 1 st quartile and the 4th quartile, respectively. Of admissions, $97.7 \%(\mathrm{n}=2193)$ were on weekdays and $80.21 \%(\mathrm{n}=$ $1800)$ were an elective type. Overall, $74.3 \%(n=1667)$ of the patients had 1 or fewer comorbidities, and only $1.02 \%$ $(n=23)$ of the patients experienced 1 or more complications after the procedure with the most common being postoperative hemorrhage or infarction $(0.44 \%, \mathrm{n}=10)$ (Table 1). The annual case volume increased from 418 in
TABLE 1: Patient and hospital characteristics for 2244 patients who underwent surgical treatment for PD between 2006 and 2010 in the US*

\begin{tabular}{|c|c|}
\hline Characteristic & Value \\
\hline \multicolumn{2}{|l|}{ age in yrs } \\
\hline mean $\pm \mathrm{SD}$ & $64.22 \pm 9.803$ \\
\hline median (range) & $65.00(23-92)$ \\
\hline interquartile range & $58-71$ \\
\hline \multicolumn{2}{|l|}{ sex } \\
\hline male & $1523(68.7)$ \\
\hline female & $694(31.3)$ \\
\hline \multicolumn{2}{|l|}{ race } \\
\hline white & $1454(64.8)$ \\
\hline black & $20(0.9)$ \\
\hline Hispanic & $133(5.9)$ \\
\hline Asian or Pacific Islander & $44(2.0)$ \\
\hline Native American & $5(0.2)$ \\
\hline other & $75(3.3)$ \\
\hline missing & $513(22.9)$ \\
\hline \multicolumn{2}{|l|}{ primary expected payer (uniform) } \\
\hline Medicare & $1405(62.8)$ \\
\hline Medicaid & $44(2.0)$ \\
\hline private & $718(32.1)$ \\
\hline self-pay & $14(0.6)$ \\
\hline no charge & $3(0.1)$ \\
\hline other & $52(2.3)$ \\
\hline \multicolumn{2}{|c|}{ median household income quartile for pt's ZIP code } \\
\hline$\$ 1-38,999$ & $411(18.8)$ \\
\hline$\$ 39,000-47,999$ & $561(25.6)$ \\
\hline$\$ 48,000-62,999$ & $595(27.2)$ \\
\hline$\geq \$ 63,000$ & $622(28.4)$ \\
\hline \multicolumn{2}{|l|}{ hospital bedsize } \\
\hline small & $139(6.3)$ \\
\hline medium & $156(7.1)$ \\
\hline large & $1907(86.6)$ \\
\hline \multicolumn{2}{|l|}{ hospital vol } \\
\hline high & $1453(64.8)$ \\
\hline low & 791 (35.2) \\
\hline \multicolumn{2}{|l|}{ comorbidity index } \\
\hline high & $577(25.7)$ \\
\hline low & $1667(74.3)$ \\
\hline \multicolumn{2}{|l|}{ treatment period } \\
\hline 2006 & $418(18.6)$ \\
\hline 2007 & $368(16.4)$ \\
\hline 2008 & $418(18.6)$ \\
\hline 2009 & $463(20.6)$ \\
\hline 2010 & $577(25.7)$ \\
\hline \multicolumn{2}{|l|}{ discharge } \\
\hline routine & 1972 (87.9) \\
\hline OTR & $272(12)$ \\
\hline
\end{tabular}

(continued) 
TABLE 1: Patient and hospital characteristics for 2244 patients who underwent surgical treatment for PD between 2006 and 2010 in the US (continued)*

\begin{tabular}{|c|c|}
\hline Characteristic & Value \\
\hline \multicolumn{2}{|l|}{ mean LOS in days } \\
\hline Northeast & 1.85 \\
\hline Midwest & 2.31 \\
\hline South & 1.69 \\
\hline West & 1.74 \\
\hline overall & 1.83 \\
\hline \multicolumn{2}{|l|}{ inhospital mortality } \\
\hline Northeast & $2(0.55)$ \\
\hline Midwest & $0(0)$ \\
\hline South & $0(0)$ \\
\hline West & $2(0.26)$ \\
\hline overall & $4(0.17)$ \\
\hline \multicolumn{2}{|l|}{ overall complications } \\
\hline $\begin{array}{l}\text { neurological including postop hemorrhage/ } \\
\text { infarction }\end{array}$ & $10(0.44)$ \\
\hline $\begin{array}{l}\text { hemorrhage/hematoma/seroma complicating a } \\
\text { procedure }\end{array}$ & $4(0.17)$ \\
\hline $\begin{array}{l}\text { mechanical complication of nervous system } \\
\text { device/implant }\end{array}$ & $5(0.2)$ \\
\hline infection due to nervous system implant & $1(0.4)$ \\
\hline removal of intracranial neurostimulator lead & $3(0.13)$ \\
\hline total & $23(1.02)$ \\
\hline \multicolumn{2}{|l|}{ procedure type } \\
\hline thalamotomy & $7(0.3)$ \\
\hline pallidotomy & $6(0.3)$ \\
\hline implantation of intracranial neurostimulator & $2228(99.3)$ \\
\hline
\end{tabular}

* Values are number of patients (\%) unless otherwise noted. pt = patient.

2006 to 577 in 2010 (Fig. 1). The majority of the patients was discharged to home or self-care $(87.9 \%, \mathrm{n}=1972)$. The inpatient hospital mortality rate was only $0.17 \%$ (n $=4$ ) during the study period with none in the years 2008 and 2010 and 1 each in the years 2006 and 2009.

Patient Characteristics and Adverse Discharge Disposition (OTR)

Overall, $12 \%(\mathrm{n}=272)$ of the patients had an adverse discharge disposition. In univariate analysis, advanced patient age at admission ( $>65$ years) and female sex showed a positive correlation with the adverse discharge disposition $(p<0.05)$. In contrast, private insurance, lower comorbidity index, and absence of complications showed a negative correlation with the adverse discharge disposition (OTR) $(\mathrm{p}<0.05)$. Adverse discharge disposition was 1.5 times more likely in patients with a high comorbidity index (OR 1.508 [95\% CI 1.148-1.98], p = 0.004) and 3 times more likely in patients with complications (OR 3.155 [95\% CI 1.202-8.279], $\mathrm{p}=0.033$ ) (Table 2).

In multivariate regression analysis, elderly patients were more likely to have adverse outcomes when com-

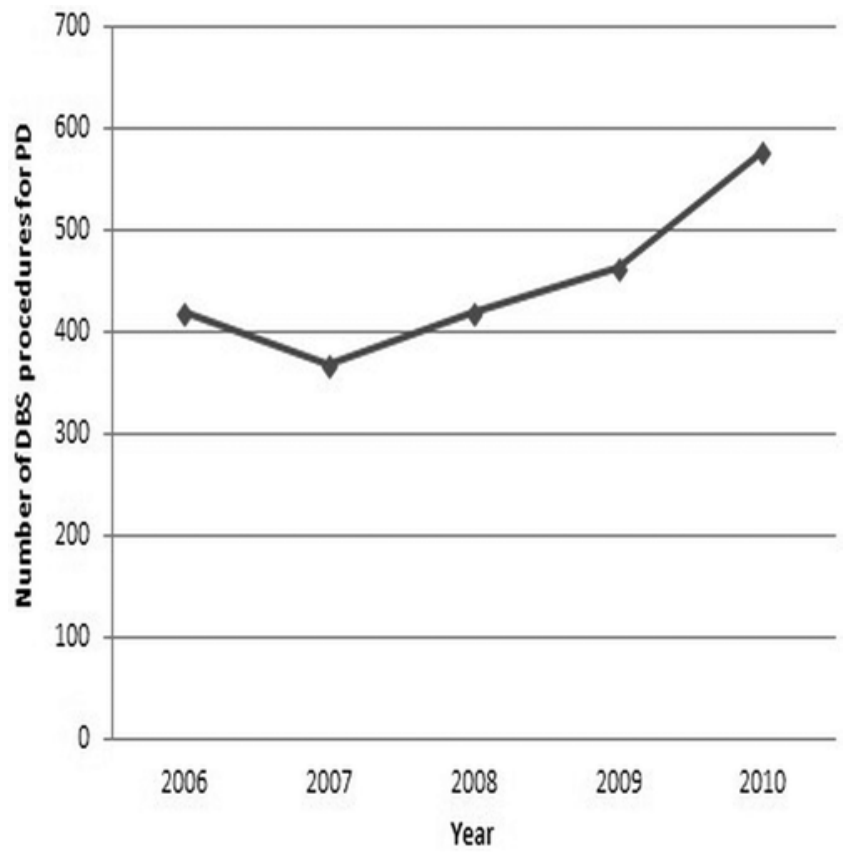

FIG. 1. Graph showing the trends in the number of DBS surgeries for PD from 2006 to 2010.

pared with those younger than 65 years of age (OR 1.596 [95\% CI 1.112-2.289], $\mathrm{p}=0.011$ ). Sex, race, high comorbidity index, presence of complications, type of insurance, and median household income did not show significant correlation with OTR discharges in the multivariate model (Table 3). Of these factors, only advanced age was an independent predictor of OTR discharge dispositions (Fig. 2).

\section{Hospital Characteristics and Adverse Discharge Disposition}

The surgical procedures for PD were performed at 150 hospitals by 91 identified surgeons in the database during the study period. The majority of the procedures were performed at centers with high case volume $(64.8 \%$, $\mathrm{n}=1453)$, at academic institutions $(85.33 \%, \mathrm{n}=1915)$, in urban areas $(\mathrm{n}=2158,96.16 \%)$, and at hospitals with a large bedsize $(86.6 \%, \mathrm{n}=1907)$ in the West or South (Table 4).

Hospitals with a large bedsize showed a positive correlation with the favorable discharge disposition compared with the hospitals with medium or smaller bedsize $(\mathrm{p}<0.05)$. Similarly, hospitals with high case volumes had a small number of patients with OTR discharge disposition than those with low case volumes (9.8\% vs $16.3 \%, \mathrm{p}<0.001)$. Moreover, hospitals with a higher annual caseload were 3.5 times more likely to have a favorable discharge disposition than those with lower case volume (OR 3.543 [95\% CI 1.781-7.048], p < 0.001), and this was an independent predictor of adverse discharge disposition (Table 5). There was no significant difference in the inhospital mortality rates between the high- (4th quartile) and low-volume centers $(0.2 \%$ vs $0.12 \%, \mathrm{p}=$ 0.734). Any complication (neurological, mechanical/de- 
Outcomes after DBS surgery for Parkinson's disease

TABLE 2: Patient and demographic factors affecting adverse discharge disposition (OTR) in patients who underwent surgical treatment for PD

\begin{tabular}{|c|c|c|c|c|}
\hline \multirow[b]{2}{*}{ Variable } & \multicolumn{2}{|c|}{ No. of Pts } & \multirow[b]{2}{*}{ Total } & \multirow[b]{2}{*}{ p Value* } \\
\hline & Routine & OTR & & \\
\hline age in yrs & & & & $<0.001$ \\
\hline$\leq 65$ & 1027 & 99 & 1126 & \\
\hline$>65$ & 935 & 173 & 1108 & \\
\hline $\operatorname{sex}$ & & & & 0.009 \\
\hline male & 1355 & 168 & 1523 & \\
\hline female & 592 & 102 & 694 & \\
\hline race & & & & 0.176 \\
\hline white & 1272 & 182 & 1454 & \\
\hline black & 15 & 5 & 20 & \\
\hline other & 229 & 28 & 257 & \\
\hline primary expected payer (uniform) & & & & $<0.001$ \\
\hline Medicare & 1200 & 205 & 1405 & \\
\hline Medicaid & 39 & 5 & 44 & \\
\hline private & 666 & 52 & 718 & \\
\hline self-pay & 11 & 3 & 14 & \\
\hline no charge & 3 & 0 & 3 & \\
\hline other & 47 & 5 & 52 & \\
\hline median household income quartile for pt ZIP code & & & & 0.736 \\
\hline$\$ 1-38,999$ & 360 & 51 & 411 & \\
\hline$\$ 39,000-47,999$ & 485 & 76 & 561 & \\
\hline$\$ 48,000-62,999$ & 526 & 69 & 595 & \\
\hline$\geq \$ 63,000$ & 549 & 73 & 622 & \\
\hline complications & & & & 0.033 \\
\hline no & 1958 & 266 & 1958 & \\
\hline yes & 14 & 6 & 14 & \\
\hline comorbidity index & & & & 0.004 \\
\hline low & 1485 & 182 & 1667 & \\
\hline high & 487 & 90 & 577 & \\
\hline
\end{tabular}

* Values in boldface are statistically significant.

vice related, hematoma, infectious and requiring device removal) occurred in $1.2 \%(n=18)$ of 1453 procedures performed at hospitals in the 4th quartile compared with $0.97 \%(\mathrm{n}=1)$ of 103 procedures performed at hospitals in the 1st quartile $(\mathrm{p}=0.90)$. The hospital control, teaching status, hospital region, day of admission, admission type, and location of the hospital had no influence on the adverse discharge disposition ( $p>0.05)$. In adjusted multivariate analysis, higher hospital caseload and government hospitals showed a significant correlation with favorable discharge outcomes. Similar to the effect of hospital caseload, the surgeon annual caseload had a positive correlation with the favorable discharge disposition. The procedures performed by surgeons with higher annual caseloads (4th quartile) had a better discharge disposition than those with lower case volumes (1st quartile) $(\mathrm{p}=0.006)$; however, after adjustment for region, hospital bedsize, hospital location, admission day and type, there was no influence of surgeon caseload on discharge disposition. There was no significant correlation between surgeon case volume and complication rates $(\mathrm{p}=0.58)$ or surgeon case volume and inpatient mortality rates $(\mathrm{p}=$ 0.70) (Figs. 3 and 4).

\section{Costs of Hospitalization and LOS}

The mean total cost of hospitalization increased from $\$ 56,534.30$ in 2006 to $\$ 59,875.27$ in 2010 with the overall mean expenditure of $\$ 61,362.93 \pm \$ 36,016.21$. Table 6 shows a difference of means in the total expenditure in different years that was found to be statistically significant ( $p<0.001)$ using the Kruskal-Wallis test. The difference in mean hospital charges between hospitals with larger case volumes versus smaller case volumes $(\$ 57,079.44$ vs $\$ 69,531.83)$, larger bedsize versus smaller bedsize hospitals $(\$ 62,262.62$ vs $\$ 64,513.63)$, government versus private $(\$ 61,239.29$ vs $\$ 65,849.29)$, teaching versus nonteaching hospitals $(\$ 60,990.98$ vs $\$ 66,633.65)$, urban versus rural location $(\$ 61,830.09$ vs $\$ 57,461.95)$, and elective versus nonelective admission type $(\$ 56,568.88$ vs $\$ 82,571.73)$ was significant $(\mathrm{p}<0.05)$ (Table 6). Among hospital re- 
TABLE 3: Multivariate binary logistic regression analysis showing various patients and demographic factors affecting the adverse discharge disposition (OTR)

\begin{tabular}{|c|c|c|c|c|c|}
\hline \multirow[b]{2}{*}{ Variable* } & \multirow[b]{2}{*}{ B Value } & \multirow[b]{2}{*}{ p Value } & \multirow[b]{2}{*}{ OR } & \multicolumn{2}{|c|}{$95 \% \mathrm{Cl}$} \\
\hline & & & & Lower Limit & Upper Limit \\
\hline age ( $\leq 65 \mathrm{yrs})$ & 0.467 & 0.011 & 1.596 & 1.112 & 2.289 \\
\hline sex (female) & 0.310 & 0.048 & 1.364 & 1.003 & 1.856 \\
\hline \multicolumn{6}{|l|}{ race compared w/ white } \\
\hline black & 0.710 & 0.230 & 2.035 & 0.638 & 6.495 \\
\hline other & -0.067 & 0.768 & 0.935 & 0.599 & 1.460 \\
\hline \multicolumn{6}{|c|}{ primary expected payer compared w/ private insurance } \\
\hline Medicare & 0.278 & 0.183 & 1.320 & 0.877 & 1.989 \\
\hline Medicaid & 0.209 & 0.710 & 1.233 & 0.410 & 3.706 \\
\hline \multicolumn{6}{|c|}{$\begin{array}{l}\text { median household income quartile for pt ZIP code } \\
<\$ 38,999 \text { compared w/ others }\end{array}$} \\
\hline$\$ 39,000-47,999$ & 0.105 & 0.653 & 1.111 & 0.701 & 1.760 \\
\hline$\$ 48,000-62,999$ & -0.013 & 0.955 & 0.987 & 0.624 & 1.560 \\
\hline$\$ \geq 63,000$ & -0.034 & 0.885 & 0.967 & 0.612 & 1.527 \\
\hline complications (yes) & 1.052 & 0.083 & 2.862 & 0.871 & 9.401 \\
\hline comorbidity index (high) & 0.270 & 0.103 & 1.310 & 0.947 & 1.813 \\
\hline
\end{tabular}

gions, the West was associated with the highest cost of hospitalization whereas the South and the Northeast had the lowest total hospital charges $(\$ 68,698.52, \$ 54,081.31$, and $\$ 58,678.28, \mathrm{p}<0.001)$. Day of admission, higher comoribidity index, and occurrence of complications had no effect on the mean total hospital charges $(p>0.05)$. Similar to the hospital case volume, surgeon case volume had an influence on the total hospital charges while those in the 4th quartile had the lowest cost of hospitalization
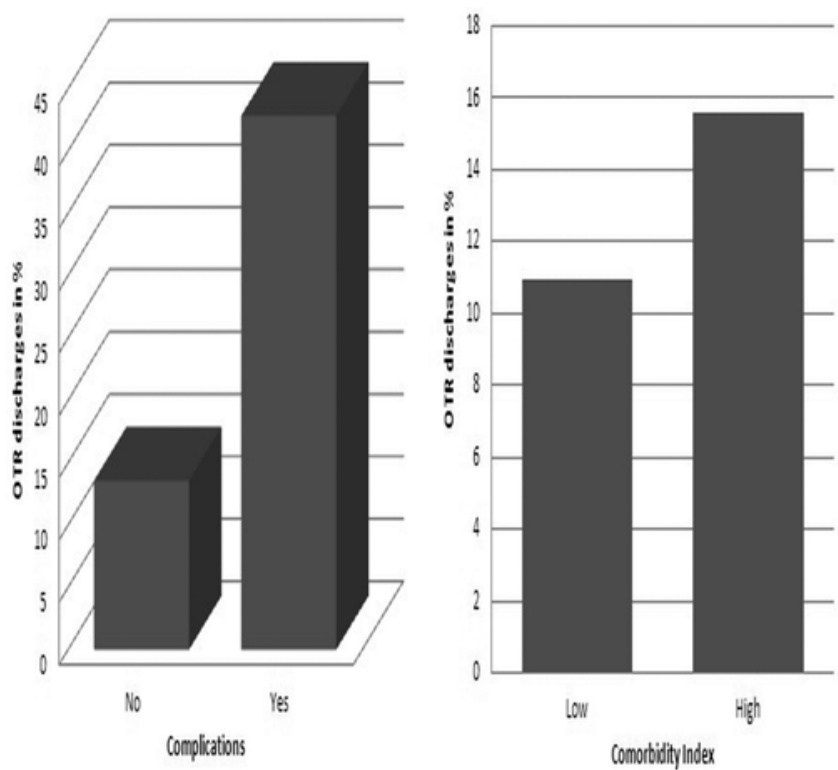

FIG. 2. Bar graphs showing the impact of postoperative complications and high comorbidity index on adverse discharge disposition after DBS surgery for PD from 2006 to 2010. compared with those in the 1st quartile (\$51,922.31 vs $\$ 71,336.32, \mathrm{p}<0.001)$. As mentioned in Methods, this variable was further dichotomized into high $(>75$ th percentile) and low cost of hospitalization. None of the patient factors (age, sex, race, type of insurance, median income, complications, and comorbidities) had an influence on the total cost of hospitalization in both univariate and multivariate analyses (Table 7). Among hospital factors, the following factors were found to be independent predictors of higher hospital cost: hospitals with a lower case volume (OR $0.093, \mathrm{p}<0.001)$, private hospitals (OR 11.027, $\mathrm{p}<0.001$ ), nonteaching hospitals (OR 3.139, $\mathrm{p}=$ 0.003 ), and hospitals in the West compared with those in the Northeast and Midwest (OR $1.885[\mathrm{p}=0.033]$ and OR 2.897 [ $p=0.031$ ], respectively). Nonelective admissions were associated with higher total hospital charges than elective admissions ( $p<0.001)$, whereas the day of admission and the hospital bedsize had no influence on the total cost of hospitalization ( $p=0.127$ and 0.145 , respectively). Surgeons with case volumes in the 4th quartile had a lower cost incurred to the hospitals than those in the lower quartiles ( $<<0.001)$ (Tables 8 and 9 and Fig. 5). from 2.03 days in 2006 to 1.55 days in 2010 (Fig. 6). The mean LOS was highest in the Midwest (2.31 days) with the overall mean stay of 1.83 days. Surprisingly, the annual hospital caseload had no influence on hospital LOS $(\mathrm{p}=0.990)$, whereas hospitals with smaller bedsize and surgeons with higher annual case volume were associated with a shorter hospital stay than hospitals with larger bedsize and surgeons with the lower annual case volume $(\mathrm{p}=0.016$ and $\mathrm{p}=0.003$, respectively). Similarly private hospitals had a shorter hospital stay than government hospitals $(\mathrm{p}<0.001)$. 
Outcomes after DBS surgery for Parkinson's disease

TABLE 4: Hospital factors affecting adverse discharge disposition (OTR) following surgical management of patients with PD

\begin{tabular}{|c|c|c|c|c|}
\hline \multirow[b]{2}{*}{ Variable } & \multicolumn{2}{|c|}{ No. of Pts } & \multirow[b]{2}{*}{ Total } & \multirow[b]{2}{*}{ p Value* } \\
\hline & Routine & OTR & & \\
\hline hospital bedsize & & & & 0.022 \\
\hline small & 121 & 18 & 139 & \\
\hline medium & 126 & 30 & 156 & \\
\hline large & 1684 & 223 & 1907 & \\
\hline hospital vol & & & & $<0.001$ \\
\hline high & 1310 & 143 & 1453 & \\
\hline low & 662 & 129 & 791 & \\
\hline \multicolumn{5}{|l|}{ hospital control } \\
\hline government & 1731 & 236 & 1967 & 0.207 \\
\hline private & 200 & 35 & 235 & \\
\hline teaching status of hospital & & & & 0.101 \\
\hline nonteaching & 243 & 44 & 287 & \\
\hline teaching & 1688 & 227 & 1915 & \\
\hline location of hospital & & & & 0.483 \\
\hline rural & 37 & 7 & 44 & \\
\hline urban & 1894 & 264 & 2158 & \\
\hline hospital region & & & & 0.115 \\
\hline Northeast & 310 & 53 & 363 & \\
\hline Midwest & 293 & 50 & 343 & \\
\hline South & 702 & 88 & 790 & \\
\hline West & 667 & 81 & 748 & \\
\hline admission day & & & & 0.665 \\
\hline weekday & 1928 & 265 & 2193 & \\
\hline weekend & 44 & 7 & 51 & \\
\hline admission status & & & & 0.417 \\
\hline elective & 1587 & 213 & 1800 & \\
\hline nonelective & 385 & 59 & 444 & \\
\hline surgeon case vol & & & & 0.006 \\
\hline 1st quartile & 18 & 7 & 25 & \\
\hline 2nd quartile & 52 & 8 & 60 & \\
\hline 3rd quartile & 106 & 21 & 127 & \\
\hline 4th quartile & 595 & 63 & 658 & \\
\hline
\end{tabular}

* Values in boldface are statistically significant.

\section{Discussion}

In our study, we analyzed a representative sample of 2244 patients who underwent surgical treatment for PD in the US from 2006 to 2010. In the previous study, Eskandar et al. reported the practice patterns, short-term outcomes, and total hospital charges after surgery for PD in the US from 1996 to $2000 .^{10}$ Similarly Lad et al. studied the socioeconomic trends in DBS surgery for all indications using the NIS from 1993 to $2006 .{ }^{14}$ However, no study has focused on the demographic and hospitalrelated factors that can affect the discharge disposition and the total cost of hospitalization in the recent years.

The number of patients who underwent surgical treatment for PD in the US increased from 1761 in
1996-2000 to 2244 in 2006-2010 with an increase in the number of reporting hospitals and surgeons from 71 and 61 to 150 and 91 , respectively. ${ }^{10}$ The choice of surgical procedure between ablative (pallidotomy and thalamotomy) and neurostimulation for PD underwent a paradigm shift in the last decade. In 1996 all procedures performed for PD were ablative in nature and changed to DBS (88\% of the procedures) in $2000 . .^{10}$ In our study, $99.3 \%$ of the procedures performed for PD were DBS and only $0.6 \%$ of the procedures were ablative in nature.The incidence of adverse discharge disposition (OTR) had increased from $8.1 \%$ in $1996-2000$ to $12 \%$ in $2006-2010$; this is in contrast to the inhospital mortality rates, which had decreased from $0.2 \%$ to $0.17 \%$ during the same treatment periods..$^{10}$ Similarly, compared with the results of an ear- 
TABLE 5: Multivariate binary logistic regression analysis showing various hospital factors affecting adverse discharge disposition (OTR)

\begin{tabular}{|c|c|c|c|c|c|}
\hline \multirow[b]{2}{*}{ Variable* } & \multirow[b]{2}{*}{ B Value } & \multirow[b]{2}{*}{ p Value $†$} & \multirow[b]{2}{*}{ OR } & \multicolumn{2}{|c|}{$95 \% \mathrm{Cl}$} \\
\hline & & & & Lower Limit & Upper Limit \\
\hline \multicolumn{6}{|l|}{ hospital bedsize compared w/ small } \\
\hline medium & 0.307 & 0.660 & 1.359 & 0.347 & 5.325 \\
\hline large & 0.678 & 0.228 & 1.971 & 0.654 & 5.935 \\
\hline high hospital vol (yes) & 1.265 & $<0.001$ & 3.543 & 1.781 & 7.048 \\
\hline government hospital control & -2.016 & 0.004 & 0.133 & 0.034 & 0.524 \\
\hline teaching status of hospital (yes) & -0.352 & 0.469 & 0.703 & 0.271 & 1.826 \\
\hline urban location of hospital (yes) & -0.058 & 0.932 & 0.944 & 0.252 & 3.530 \\
\hline \multicolumn{6}{|l|}{ hospital region compared w/ West } \\
\hline Northeast & -0.527 & 0.135 & 0.591 & 0.296 & 1.179 \\
\hline Midwest & -0.493 & 0.358 & 0.611 & 0.213 & 1.747 \\
\hline South & -0.352 & 0.283 & 0.703 & 0.370 & 1.338 \\
\hline weekend admission day (yes) & 1.625 & 0.089 & 5.077 & 0.781 & 32.995 \\
\hline elective admission status (yes) & 0.616 & 0.197 & 1.851 & 0.726 & 4.720 \\
\hline \multicolumn{6}{|c|}{ surgeon case vol compared $w /$ those in the 4 th quartile } \\
\hline 1st quartile & 0.887 & 0.089 & 2.429 & 0.874 & 6.747 \\
\hline 2nd quartile & -0.249 & 0.607 & 0.780 & 0.303 & 2.009 \\
\hline 3rd quartile & 0.441 & 0.229 & 1.554 & 0.758 & 3.187 \\
\hline
\end{tabular}

lier study of surgically treated patients with PD in the US between 1996 and 2000, the incidence of postoperative neurological complications, hematoma complicating the procedure, and mechanical complications decreased from $1.5 \%, 0.5 \%$, and $1 \%$ to $0.44 \%, 0.17 \%$, and $0.2 \%$, re-
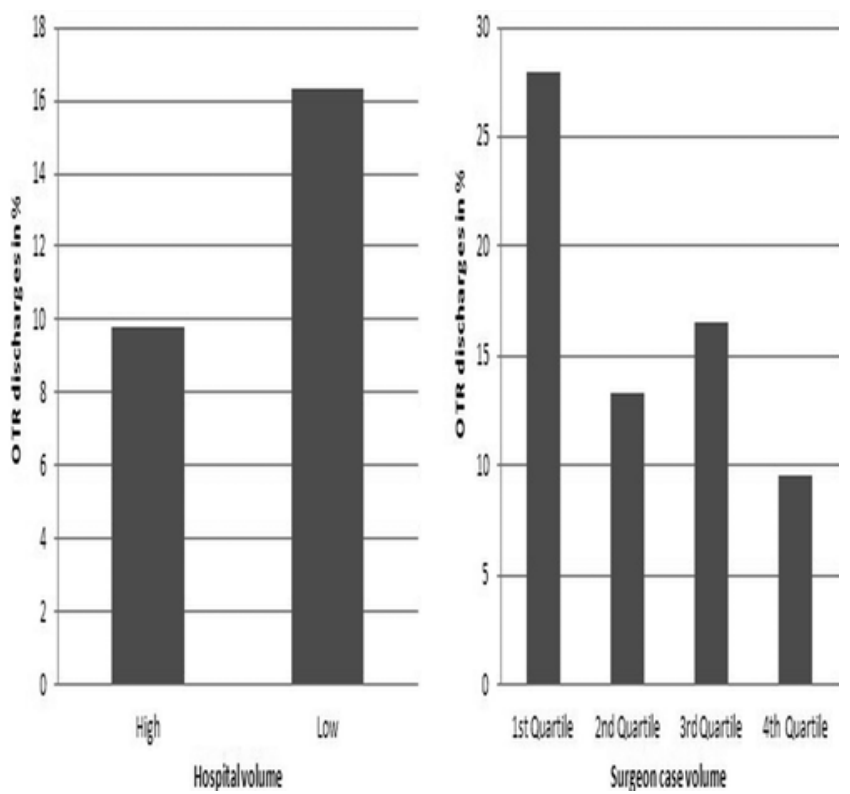

FIG. 3. Bar graphs showing the impact of hospital volumes (high vs low) and surgeon case volumes (by quartiles, 1st quartile, 1 case; 2 nd quartile, 2 or 3 cases; 3rd quartile, $4-12$ cases; and 4th quartile, 13 or more cases) on adverse discharge disposition following DBS surgery for PD from 2006 to 2010. spectively. Pilitis et al. reported that the inhospital complications following DBS for PD or essential tremors in the US from 1998 to 2007 had decreased from $3.8 \%$ to $2.8 \% .^{22}$ In our study, only 1 patient $(0.4 \%)$ developed DBS electrode/implant-related infection. However, since there is always a delay between implant surgery and the onset of infection, NIS-based studies may underestimate the

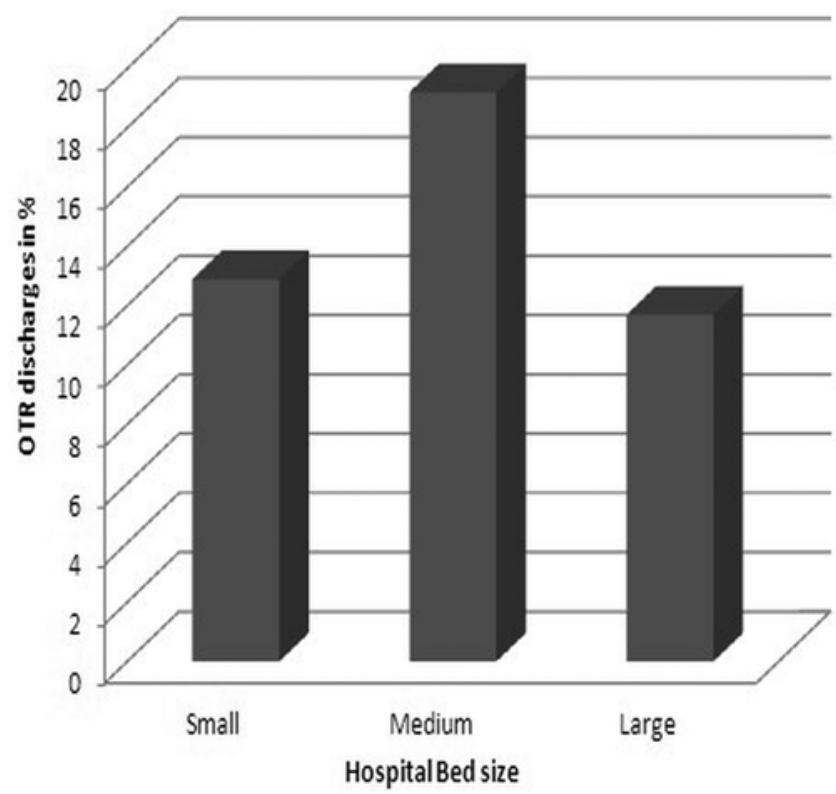

FIG. 4. Bar graphs showing the impact of hospital bedsize on adverse discharge disposition after DBS surgery for PD from 2006 to 2010. 
TABLE 6: Difference between the mean total hospital charges among various groups (Kruskal-Wallis test)

\begin{tabular}{|c|c|c|}
\hline Variable & Mean Total Charges (\$) & p Value* \\
\hline year of treatment & & $<0.001$ \\
\hline 2006 & $56,534.30$ & \\
\hline 2007 & $65,072.41$ & \\
\hline 2008 & $62,867.82$ & \\
\hline 2009 & $63,271.76$ & \\
\hline 2010 & $59,875.27$ & \\
\hline complications & & 0.886 \\
\hline yes & $59,109.32$ & \\
\hline no & $61,382.55$ & \\
\hline comorbidity index & & 0.865 \\
\hline low & $61,420.86$ & \\
\hline high & $61,193.16$ & \\
\hline hospital bedsize & & 0.002 \\
\hline small & $64,513.63$ & \\
\hline medium & $53,006.67$ & \\
\hline large & $62,262.62$ & \\
\hline hospital control & & 0.020 \\
\hline government & $61,239.29$ & \\
\hline private & $65,849.29$ & \\
\hline teaching status of hospital & & $<0.001$ \\
\hline nonteaching & $66,633.65$ & \\
\hline teaching & $60,990.98$ & \\
\hline location of hospital & & 0.001 \\
\hline rural & $57,461.95$ & \\
\hline urban & $61,830.09$ & \\
\hline hospital region & & $<0.001$ \\
\hline Northeast & $58,678.28$ & \\
\hline Midwest & $65,812.40$ & \\
\hline South & $54,081.31$ & \\
\hline West & $68,698.52$ & \\
\hline admission day & & 0.901 \\
\hline weekday & $61,492.37$ & \\
\hline weekend & $55,556.79$ & \\
\hline admission type & & $<0.001$ \\
\hline elective & $56,568.88$ & \\
\hline nonelective & $82,571.73$ & \\
\hline surgeon case vol & & $<0.001$ \\
\hline 1st quartile & $71,336.32$ & \\
\hline 2nd quartile & $63,347.05$ & \\
\hline 3rd quartile & $63,590.17$ & \\
\hline 4th quartile & $51,922.31$ & \\
\hline hospital case vol & & $<0.001$ \\
\hline 1st quartile & $69,531.83$ & \\
\hline 2nd quartile & $66,292.80$ & \\
\hline 3rd quartile & $70,099.02$ & \\
\hline 4th quartile & $57,079.44$ & \\
\hline
\end{tabular}

* Values in boldface are statistically significant. true incidence of this complication as the outcomes can be measured only at the time of discharge. Similarly, the mean LOS after DBS for PD had decreased from 2 days in 1996-2000 to 1.83 days in 2006-2010..$^{10}$ These results reflect the advances in neurosurgical techniques, imaging modalities, intraoperative monitoring, and improvement in postoperative care.

\section{Patient Factors and Outcome}

Eskandar et al. showed that the young, male Caucasian patients with private insurance and higher median household income and fewer comorbidities were more likely to undergo implantation of neurostimulators than lesion therapy for PD in the US from 1996 to $2000 . .^{10}$ However, in our study from 2006 to 2010, DBS was performed equally in patients younger or older than 65 years of age $(50.17 \%$ vs $49.37 \%)$, and the majority of patients had nonprivate insurance. Apart from these 2 variables the rest of the variables were in concordance with the previous study. Similarly Lad et al. showed that DBS was performed in $56 \%$ of males compared with $43 \%$ of females in the US from 1993 to $2006 \cdot{ }^{14}$ In another study, patients with PD who underwent DBS in the 2007 in the US were older and had significantly higher comorbidities. ${ }^{22}$ These findings reflect a change in the pattern of DBS surgery for PD in the US, which initially used to be performed in younger healthier patients. The fact that the majority of DBS procedures for PD were performed in male Caucasian patients with a higher median income can be attributed to the biases by the treating physician or the patients themselves. ${ }^{10}$ In our study, favorable discharge disposition was seen in young, male patients with a private insurance, low comorbidity index, and without postoperative complications. In our study, inhospital mortality was seen in $8.69 \%$ of the patients with complications and in $0.09 \%$ of the patients without complications, which was statistically significant $(\mathrm{p}<0.001)$. Therefore, appropriate measures to avoid them and prompt diagnosis and management of these complications cannot be overemphasized. Apart from these demographic factors, other described factors that can affect the outcome such as preoperative neurological status cannot be evaluated due to the limitations of using the NIS database.

\section{Hospital Factors and Outcome}

Similar to other studies, the majority of DBS procedures for PD were performed at large, high-volume, urban, and academic centers. ${ }^{10,14,22}$ However, contrary to the finding that $75.2 \%$ of the total DBS procedures in the US were performed at private nonprofit centers in 2006, the majority $(87.6 \%)$ of the DBS procedures for PD were carried out at government-owned hospitals from 2006 to 2010 in the US. ${ }^{14}$ The concept of "volume effect" has been explained in earlier studies on the NIS and was extensively investigated for carotid endarterectomy and aneurysm surgery. ${ }^{2-4,10,11,13,19,23}$ Various studies have shown superior outcomes at high-volume centers and large bedsize hospitals. In concordance with this concept, our study showed that the hospitals with large bedsize and high case volumes were 1.7 and 3.5 times more likely to have 
TABLE 7: Patient and demographic factors affecting total hospital charges after surgical treatment of PD

\begin{tabular}{|c|c|c|c|c|}
\hline \multirow[b]{2}{*}{ Variable } & \multicolumn{2}{|l|}{ No. of Pts } & \multirow[b]{2}{*}{ Total } & \multirow[b]{2}{*}{ p Value } \\
\hline & High Cost (>75th percentile) & Low Cost & & \\
\hline age in yrs & & & & 0.136 \\
\hline$\leq 65$ & 262 & 841 & 1103 & \\
\hline$>65$ & 282 & 808 & 1090 & \\
\hline $\operatorname{sex}$ & & & & 0.209 \\
\hline male & 375 & 1116 & 1491 & \\
\hline female & 161 & 526 & 687 & \\
\hline race & & & & 0.643 \\
\hline white & 373 & 1052 & 1425 & \\
\hline black & 5 & 14 & 19 & \\
\hline other & 72 & 176 & 248 & \\
\hline primary expected payer (uniform) & & & & 0.307 \\
\hline Medicare & 352 & 1031 & 1383 & \\
\hline Medicaid & 11 & 33 & 44 & \\
\hline private & 165 & 533 & 698 & \\
\hline self-pay & 6 & 8 & 14 & \\
\hline no charge & 2 & 1 & 3 & \\
\hline other & 13 & 39 & 52 & \\
\hline median household income quartile for pt ZIP code & & & & 0.735 \\
\hline$\$ 1-38,999$ & 102 & 305 & 407 & \\
\hline$\$ 39,000-47,999$ & 144 & 410 & 554 & \\
\hline$\$ 48,000-62,999$ & 136 & 449 & 585 & \\
\hline$\geq \$ 63,000$ & 152 & 448 & 600 & \\
\hline complications & & & & 0.535 \\
\hline no & 545 & 1637 & 2182 & \\
\hline yes & 5 & 14 & 19 & \\
\hline comorbidity index & & & & 0.99 \\
\hline low & 410 & 1231 & 1641 & \\
\hline high & 140 & 420 & 560 & \\
\hline
\end{tabular}

a favorable discharge disposition than those with small bedsize and low case volumes, respectively. However, the fact that high-volume centers are more likely to perform simultaneous bilateral implantation of DBS electrodes for PD may underestimate the total number of procedures performed and overestimate the risk of adverse outcomes per procedure at such centers. Similarly, transfer of postoperative patients to inpatient rehabilitation care may skew adverse discharge disposition to favor those hospitals where such facilities are available and cannot be coded separately in the NIS database. Therefore, due to the inherent limitations of the NIS database, these results should be cautiously interpreted. Similarly, surgeons with higher annual case volumes were less likely to have an adverse discharge disposition than those with lower case volumes $(p<0.05)$. These findings reinforce the fact that superior outcomes correlate with increased surgeon experience. However, the incidence of postoperative complications and inhospital mortality was not different between high- and low-volume centers or between surgeons with high- and low-volume caseloads. This in contrast to the findings of Eskandar et al., who showed that the mortality and neurological complications were more likely after surgery at low-volume centers than at highvolume centers. This difference can be attributed to the very low incidence of complications and mortality rates in recent years. There was no difference in the discharge disposition among academic versus nonacademic centers and rural versus urban hospitals. However, nonacademic centers were associated with a higher cost of hospitalization than academic centers, and there was no difference in the cost between rural and urban centers. Similar to the findings of Pilitsis et al. and Lad et al., the majority of the DBS procedures for PD were performed at hospitals in the West and South. ${ }^{14,22}$

\section{Hospital Factors and Costs}

The cost of DBS surgery for PD increased from $\$ 56,534.30$ in 2006 to $\$ 59,875.27$ in 2010 . This is in concordance with the finding of Lad et al. who reported that the hospital charges of DBS had gradually increased over a period of 14 years with a total expenditure of \$291 million in $2006 .{ }^{14}$ This may be either due to an overall increase in the cost of the technology or the hospital charges over this 


\section{Outcomes after DBS surgery for Parkinson's disease}

TABLE 8: Hospital factors affecting the total cost of hospitalization after surgical management of patients with PD

\begin{tabular}{|c|c|c|c|c|}
\hline \multirow[b]{2}{*}{ Variable } & \multicolumn{2}{|c|}{ No. of Pts } & \multirow[b]{2}{*}{ Total } & \multirow[b]{2}{*}{$\mathrm{p}$ Value } \\
\hline & High Cost (75th percentile) & Low Cost & & \\
\hline hospital bedsize & & & & 0.145 \\
\hline small & 35 & 98 & 133 & \\
\hline medium & 29 & 125 & 154 & \\
\hline large & 486 & 1386 & 1872 & \\
\hline hospital vol & & & & $<0.001$ \\
\hline high & 284 & 1134 & 1418 & \\
\hline low & 266 & 517 & 783 & \\
\hline hospital control & & & & 0.001 \\
\hline government & 468 & 1456 & 1924 & \\
\hline private & 82 & 153 & 235 & \\
\hline teaching status of hospital & & & & $<0.001$ \\
\hline nonteaching & 102 & 185 & 287 & \\
\hline teaching & 448 & 1424 & 1872 & \\
\hline location of hospital & & & & 0.490 \\
\hline rural & 9 & 35 & 44 & \\
\hline urban & 541 & 1574 & 2115 & \\
\hline hospital region & & & & $<0.001$ \\
\hline Northeast & 57 & 306 & 363 & \\
\hline Midwest & 86 & 257 & 343 & \\
\hline South & 167 & 621 & 788 & \\
\hline West & 240 & 467 & 707 & \\
\hline admission day & & & & 0.127 \\
\hline weekday & 543 & 1610 & 2153 & \\
\hline weekend & 7 & 41 & 48 & \\
\hline admission status & & & & $<0.001$ \\
\hline elective & 349 & 1449 & 1798 & \\
\hline nonelective & 201 & 202 & 403 & \\
\hline surgeon case vol & & & & $<0.001$ \\
\hline 1st quartile & 11 & 14 & 25 & \\
\hline 2nd quartile & 13 & 47 & 60 & \\
\hline 3rd quartile & 32 & 95 & 127 & \\
\hline 4th quartile & 105 & 553 & 658 & \\
\hline
\end{tabular}

* Values in boldface are statistically significant.

time period. The West had the highest and Northeast had the lowest cost of hospitalization $(\mathrm{p}<0.001)$. This finding was consistent with our previous study and can be attributed to the increased cost of living in this region. ${ }^{24}$ Nonteaching hospitals were 3.1 times $(\mathrm{p}<0.001)$ more likely to have a higher cost of hospitalization than teaching hospitals, which was in contrast to the findings of earlier studies by Taylor et al. and McGuire et al. with no difference in outcomes. ${ }^{18,26}$ Low-volume hospitals, private hospitals, and nonacademic centers in the West were the independent predictors of higher hospital charges.

\section{Advantages and Limitations of the NIS Database}

The foremost advantage of the NIS database is that it provides a comprehensive sampling of the entire US nonfederal hospital community. This database avoids the selection bias at both the individual and institutional levels and provides the largest representative sample to estimate the inpatient averages. Moreover, it also allows an accurate estimation of the hospital case volumes by providing annual discharges from each hospital. Apart from the inherent limitations of a retrospective study, there are some specific limitations of the NIS data set, such as coding errors and underreporting of events because of the inability to include outpatients. The NIS may also underestimate mortality and overestimate morbidity because the outcomes can be measured only at the time of discharge. Moreover, the duration and severity of PD before surgery as well as the severity of postoperative complications cannot be extracted from the data set. In addition, the outcome measures such as long-term functional outcome and QOL cannot be evaluated from the 
M. Sharma et al.

TABLE 9: Multivariate binary logistic regression analysis showing various hospital factors affecting total hospital charges

\begin{tabular}{|c|c|c|c|c|c|}
\hline \multirow[b]{2}{*}{ Variable* } & \multirow[b]{2}{*}{ B Value } & \multirow[b]{2}{*}{$p$ Value } & \multirow[b]{2}{*}{ OR } & \multicolumn{2}{|c|}{$95 \% \mathrm{Cl}$} \\
\hline & & & & Lower Limit & Upper Limit \\
\hline \multicolumn{6}{|l|}{ hospital bedsize compared w/ small } \\
\hline medium & -0.111 & 0.835 & 0.895 & 0.314 & 2.545 \\
\hline large & -0.255 & 0.553 & 0.775 & 0.333 & 1.800 \\
\hline high hospital vol (yes) & -2.373 & $<0.001$ & 0.093 & 0.050 & 0.175 \\
\hline government hospital control & 2.400 & $<0.001$ & 11.027 & 3.658 & 33.236 \\
\hline teaching status of hospital (yes) & 1.144 & 0.003 & 3.139 & 1.469 & 6.706 \\
\hline urban location of hospital (yes) & -21.340 & 0.998 & 0.000 & 0.000 & \\
\hline \multicolumn{6}{|l|}{ hospital region compared w/ West } \\
\hline Northeast & 0.634 & 0.033 & 1.885 & 1.052 & 3.378 \\
\hline Midwest & 1.064 & 0.031 & 2.897 & 1.104 & 7.603 \\
\hline South & 0.513 & 0.064 & 1.670 & 0.970 & 2.876 \\
\hline weekend admission day (yes) & -0.936 & 0.348 & 0.392 & 0.055 & 2.775 \\
\hline elective admission status (yes) & -0.596 & 0.158 & 0.551 & 0.241 & 1.261 \\
\hline \multicolumn{6}{|c|}{ surgeon case vol compared w/ those in the 4 th quartile } \\
\hline 1st quartile & -0.410 & 0.416 & 0.664 & 0.247 & 1.782 \\
\hline 2nd quartile & 1.203 & 0.004 & 3.331 & 1.477 & 7.510 \\
\hline 3rd quartile & 0.380 & 0.272 & 1.463 & 0.742 & 2.883 \\
\hline
\end{tabular}

* The dependent variable was a high cost of hospitalization (outcome) and the various factors mentioned were covariates.

$\dagger$ Values in boldface are statistically significant.
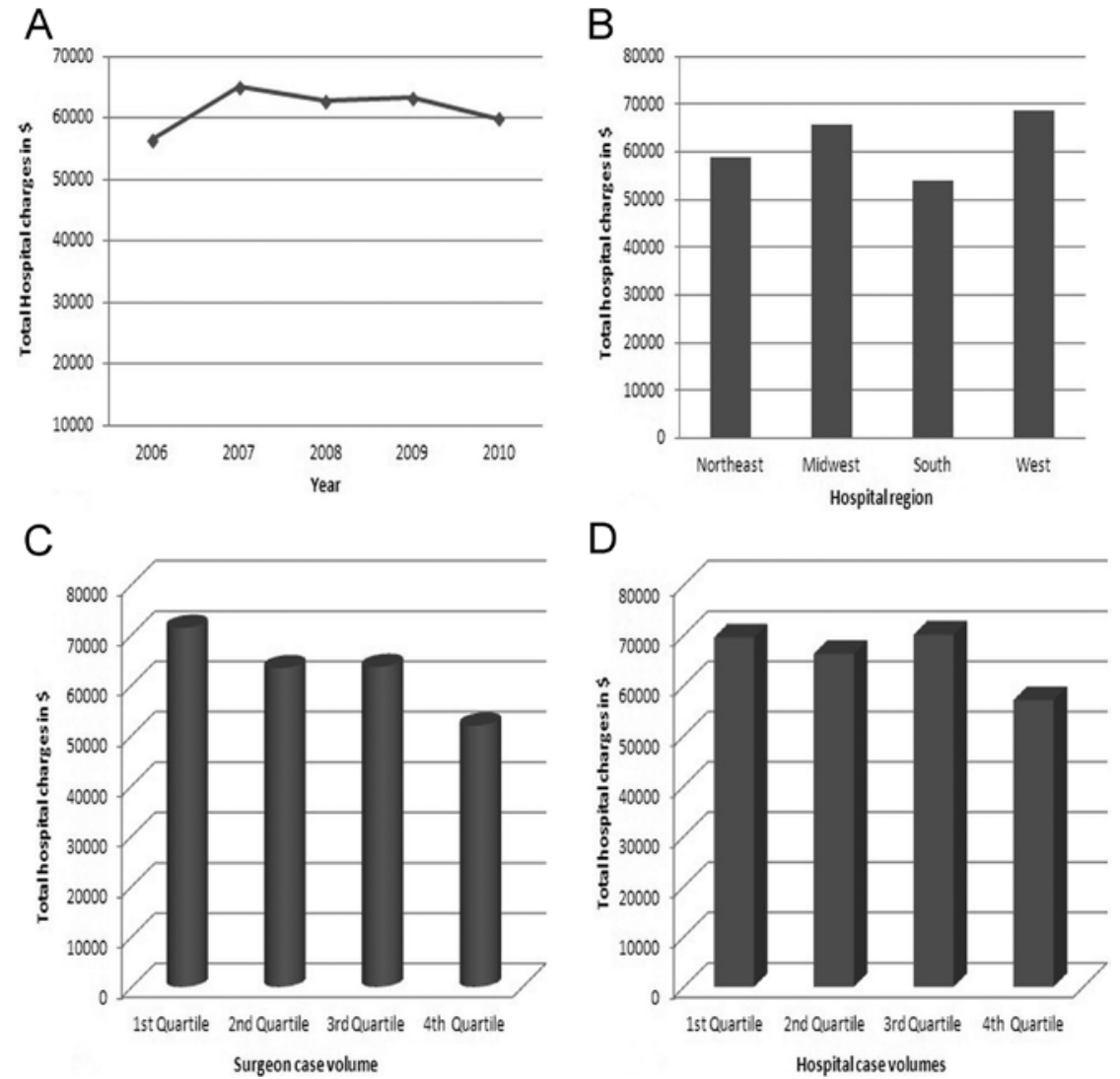

FIG. 5. Graphs showing the trends in the cost of DBS surgery for PD from 2006 to 2010 (A), impact of hospital region on total hospital charges (B), impact of surgeon case volumes (by quartiles: 1st quartile, 1 case; 2 nd quartile, 2-3 cases; 3 rd quartile, 4-12; and 4th quartile, 13 and greater) on hospital costs (C), and impact of hospital case and volumes (by quartiles: 1st quartile, 1-3; 2nd quartile, 4-9; 3rd quartile, 10-19; and 4th quartile, 20 and greater) on the total cost of hospitalization (D) after DBS surgery for PD from 2006 to 2010. 
Outcomes after DBS surgery for Parkinson's disease

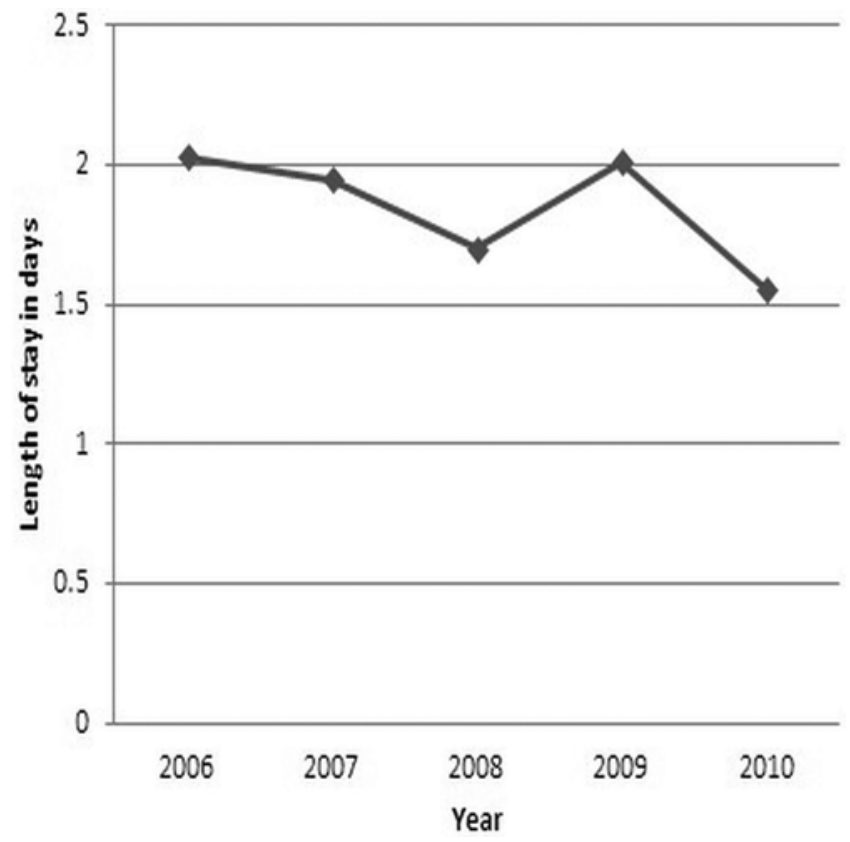

FIG. 6. Graph showing the trends in hospital stay after DBS surgery for PD from 2006 to 2010 .

NIS database, necessitating further prospective studies. Some centers perform bilateral DBS as staged procedures in separate admissions; therefore, it is possible that such patients were counted twice in this study, underestimating the LOS and cost and overestimating the total number of discharges. However, the majority of the centers perform bilateral DBS in a single admission; therefore, this limitation is unlikely to affect the outcomes. ${ }^{14}$ Nonetheless, it helps to understand the regional trends, short-term outcomes, and practice patterns in the US.

\section{Conclusions}

Our study provides an overview of the outcome, the total cost of hospitalization, and the regional variations associated with DBS surgery for PD within the US from 2006 to 2010. Elderly female patients with a nonprivate insurance and higher comorbidity index who underwent surgery at low-volume centers with a smaller bedsize and by a surgeon with a lower annual case volume and the occurrence of postoperative complications were correlated with adverse discharge dispositions. High-volume, government-owned academic centers in the Northeast with elective admissions were associated with a lower cost incurred to the hospitals. These findings suggest that there may be a disparity in terms of accessibility to the centers that are performing DBS surgery for PD. Therefore, it can be recommended that the widespread availability of this procedure across small academic centers in rural areas may not only provide an easier access to the patients but also may reduce the total cost incurred to the hospitals. Moreover, in the current scenario with the rapidly expanding novel indications for DBS, it is prudent to study the socioeconomic impact of this procedure on outcome, patient access, and the overall costs incurred.

\section{Disclosure}

The authors report no conflict of interest concerning the materials or methods used in this study or the findings specified in this paper.

Author contributions to the study and manuscript preparation include the following. Conception and design: Nanda, Sharma. Acquisition of data: Sharma. Analysis and interpretation of data: Sharma, Ambekar. Drafting the article: Sharma. Critically revising the article: all authors. Reviewed submitted version of manuscript: Wilden. Statistical analysis: Sharma, Ambekar. Administrative/ technical/material support: Nanda, Guthikonda. Study supervision: Nanda, Guthikonda.

\section{References}

1. Baker KB, Kopell BH, Malone D, Horenstein C, Lowe M, Phillips MD, et al: Deep brain stimulation for obsessive-compulsive disorder: using functional magnetic resonance imaging and electrophysiological techniques: technical case report. Neurosurgery 61 (5 Suppl 2):E367-E368, 2007

2. Bardach NS, Zhao S, Gress DR, Lawton MT, Johnston SC: Association between subarachnoid hemorrhage outcomes and number of cases treated at California hospitals. Stroke 33: $1851-1856,2002$

3. Barker FG II: Craniotomy for the resection of metastatic brain tumors in the U.S., 1988-2000: decreasing mortality and the effect of provider caseload. Cancer 100:999-1007, 2004

4. Barker FG II, Carter BS, Ojemann RG, Jyung RW, Poe DS, McKenna MJ: Surgical excision of acoustic neuroma: patient outcome and provider caseload. Laryngoscope 113:13321343,2003

5. Bucy P, Case JT: Tremor: Physiologic mechanism and abolition by surgical means. Arch Neurol Psychiatr 41:721-746, 1939

6. Charlson ME, Pompei P, Ales KL, MacKenzie CR: A new method of classifying prognostic comorbidity in longitudinal studies: development and validation. J Chronic Dis 40:373383, 1987

7. Cotzias GC, Papavasiliou PS, Gellene R: Modification of Parkinsonism-chronic treatment with L-dopa. N Engl J Med 280:337-345, 1969

8. Cotzias GC, Van Woert MH, Schiffer LM: Aromatic amino acids and modification of parkinsonism. N Engl J Med 276:374-379, 1967

9. Elixhauser A, Steiner C, Harris DR, Coffey RM: Comorbidity measures for use with administrative data. Med Care 36:827, 1998

10. Eskandar EN, Flaherty A, Cosgrove GR, Shinobu LA, Barker FG II: Surgery for Parkinson disease in the United States, 1996 to 2000: practice patterns, short-term outcomes, and hospital charges in a nationwide sample. J Neurosurg 99: 863-871, 2003

11. Hannan EL, Popp AJ, Feustel P, Halm E, Bernardini G, Waldman J, et al: Association of surgical specialty and processes of care with patient outcomes for carotid endarterectomy. Stroke 32:2890-2897, 2001

12. Henderson JM: Vagal nerve stimulation versus deep brain stimulation for treatment-resistant depression: show me the data. Clin Neurosurg 54:88-90, 2007

13. Kalkanis SN, Eskandar EN, Carter BS, Barker FG II: Microvascular decompression surgery in the United States, 1996 to 2000: mortality rates, morbidity rates, and the effects of hospital and surgeon volumes. Neurosurgery 52:1251-1262, 2003

14. Lad SP, Kalanithi PS, Patil CG, Itthimathin P, Batya S, Bronte-Stewart H, et al: Socioeconomic trends in deep brain stimulation (DBS) surgery. Neuromodulation 13:182-186, 2010

15. Laitinen LV, Bergenheim AT, Hariz MI: Leksell's postero- 


\section{Sharma et al.}

ventral pallidotomy in the treatment of Parkinson's disease. $\mathbf{J}$ Neurosurg 76:53-61, 1992

16. Laitinen LV, Bergenheim AT, Hariz MI: Ventroposterolateral pallidotomy can abolish all parkinsonian symptoms. Stereotact Funct Neurosurg 58:14-21, 1992

17. Malone DA Jr, Dougherty DD, Rezai AR, Carpenter LL, Friehs GM, Eskandar EN, et al: Deep brain stimulation of the ventral capsule/ventral striatum for treatment-resistant depression. Biol Psychiatry 65:267-275, 2009

18. McGuire KJ, Chacko AT, Bernstein J: Cost-effectiveness of teaching hospitals for the operative management of hip fractures. Orthopedics 34:e598-e601, 2011

19. O'Neill L, Lanska DJ, Hartz A: Surgeon characteristics associated with mortality and morbidity following carotid endarterectomy. Neurology 55:773-781, 2000

20. Okun MS, Vitek JL: Lesion therapy for Parkinson's disease and other movement disorders: update and controversies. Mov Disord 19:375-389, 2004

21. Pereira EA, Green AL, Nandi D, Aziz TZ: Deep brain stimulation: indications and evidence. Expert Rev Med Devices 4: 591-603, 2007

22. Pilitsis JG, Burrows A, Peters ML, Sargent J, Ng SC, Tseng JF: Changing practice patterns of deep brain stimulation in Parkinson's disease and essential tremor in the USA. Stereotact Funct Neurosurg 90:25-29, 2012

23. Solomon RA, Mayer SA, Tarmey JJ: Relationship between the volume of craniotomies for cerebral aneurysm performed at New York state hospitals and in-hospital mortality. Stroke 27:13-17, 1996
24. Sonig A, Khan IS, Wadhwa R, Thakur JD, Nanda A: The impact of comorbidities, regional trends, and hospital factors on discharge dispositions and hospital costs after acoustic neuroma microsurgery: a United States nationwide inpatient data sample study (2005-2009). Neurosurg Focus 33(3):E3, 2012

25. Steiner C, Elixhauser A, Schnaier J: The healthcare cost and utilization project: an overview. Eff Clin Pract 5:143-151, 2002

26. Taylor DH Jr, Whellan DJ, Sloan FA: Effects of admission to a teaching hospital on the cost and quality of care for Medicare beneficiaries. N Engl J Med 340:293-299, 1999

27. Thevathasan W, Cole MH, Graepel CL, Hyam JA, Jenkinson $\mathrm{N}$, Brittain JS, et al: A spatiotemporal analysis of gait freezing and the impact of pedunculopontine nucleus stimulation. Brain 135:1446-1454, 2012

28. Thevathasan W, Coyne TJ, Hyam JA, Kerr G, Jenkinson N, Aziz TZ, et al: Pedunculopontine nucleus stimulation improves gait freezing in Parkinson disease. Neurosurgery 69:12481254,2011

Manuscript submitted July 13, 2013.

Accepted August 28, 2013.

Please include this information when citing this paper: DOI: 10.3171/2013.8.FOCUS13295.

Address correspondence to: Anil Nanda, M.D., M.P.H., Department of Neurosurgery, Louisiana State University Health Sciences Center, 1501, Kings Highway, Shreveport, LA 71130. email: ananda @1suhsc.edu. 